\title{
Criteria for Designing, Building and Installing a Magnetic Minimizer
}

\author{
Raul Guerrero Torres ${ }^{1}$, Mehrab Mehrvar ${ }^{2}$ \\ ${ }^{1}$ Faculty of Engineering, University of Cartagena \\ Cartagena de Indias, Colombia \\ rguerrerotores1@unicartagena.edu.co \\ ${ }^{2}$ Department of Chemical Engineering, Ryerson University \\ 350 Victoria Street, Toronto, ON, Canada M5B 2K3 \\ mmehrvar@ryerson.ca
}

\begin{abstract}
Changes due to magnetic field actions are very small at the molecular level by permanent magnets such as Neodymium $(\mathrm{NdFeB})$. Therefore, it is not rare that different lacks of precaution result in a frequently limited global changes in the fuel and in an inefficient performance of devices using magnetic action with or without previous treatments by hydraulics changes; such as sections enlargements and/or fuel flow splitting to optimize the combustion. In addition, those changes can be attenuated or totally neutralized by variations in fuel environment conditions and/or by a defective installation of the device that give rise to unsatisfactory results or render the device completely ineffective. That is why it is necessary to precisely design magnetic devices to build them under a continuous rigorous technological control and protect them as shielding them against random electromagnetic field actions and to install them taking all the precautions to prevent environmental influences as the earth magnetic field in some instances, tending to minimize their efficiencies. There are few formal studies in the open literature on the engineering design of magnetic devices. In this study, intended to improve the efficient performance of magnetic minimizers using permanent magnets, with or without hydraulic fuel flow changes, some criteria based on theory and experience on engineering design, building and installation, based in the comparison of the most representatives magnetic devices with and without fuel hydraulic flow changes with a chosen prototype of efficiency, proved in an ADC, are presented.
\end{abstract}

Keywords: Engineering Design, Flow Splitting, Earth Magnetic Field, Neodymium Magnets, ADC.

\section{Introduction}

There are many kinds of magnetic devices patented and/or available to buy. Because of the great diversity of existing magnetic devices would be an exaggeratedly long, to describe all the different groups and subgroups of magnetic devices and compare them with the magnetic minimizer chosen as a prototype. Therefore, it is pertinent to describe only the magnetic devices that are similar in order to make a rational comparison of the prototype with others to show the advantages and disadvantages of the proved efficient prototype. Consequently, the emphasis is on the prototype and two other devices using only magnetic field action and those using the magnetic field action combined with fuel flow hydraulic changes as section enlargement and/or fuel flow splitting to ionize the fuel.

\subsection{The Prototype}

This magnetic minimizer controls the influence of conditions in its surrounding, not controlled by the similar devices of the state of the art, which often affect the effectiveness of the magnetic devices. In addition, integrates additional actions to the magnetic one, by means of a section enlargement at least $10 \mathrm{~cm}$ long and minimum diameter of $1 / 2^{\prime \prime}$, including a multi-directional flow unit, which by altering fuel flow conditions, further improves the efficiency of combustion. This consists of an antimagnetic multi-directional antimagnetic flow unit constructed of an alloy composed mainly of copper, zinc, magnesium and nickel working integrally with a pair of Neodymium magnets held at the end of the device outputting the fuel. All this elements are within an insulated conduit with a shield for protecting them against random electromagnetic fields. The device, which can be used with any type of carburetor or electronic fuel injection engine, is also provided with a small compass inserted externally at the end of the device outputting the fuel installed properly in line with the fuel conduit and in the same direction of the earth magnetic field to control its magnetic action that in some instances could count resting the neodymium magnets action on the When the fuel flows through the device, it is ionized and when combines with oxygen from the air, almost complete combustion is obtained. This implies that the reduction of pollutant 
gas emissions increases the engine efficiency and more kilometers are travelled per gallon of fuel. This prototype sizing has been, as in most of magnetic devices, the result of empirical learning through many years of test and error work.

\subsection{Devices with Only Magnetic Field Action}

There are many devices that use strong magnetic field actions of permanent magnets, such as the neodymium, to ionize fuels and improve their combustion. From this first group, it is enough to make reference only to two more similar and best-known devices ${ }^{1}$ ( The Ethos MAXPower Super Fuel and The Fuel Optimizer). They apply a magnetic field to ionize the fuel, giving rise to a more complete combustion and therefore, to have a better fuel economy for the improvement of engine efficiency and the reduction of polluting emissions. Another benefit obtained is the dissolution of carbon accumulated in the carburetor, fuel injectors, and combustion chambers, helping to clean the engine. Generally, magnets are embedded in a non-magnetic casing made of plastic, copper or aluminum material to secure them to the fuel line. Magnetic field action on the magnetic dipoles of the fuel molecules changes its orientation resulting in the rupture of clusters of molecules and consequently the ionization of the fuel and a greater area exposed to the air, improving the combination of the fuel with the oxygen of the air and consequently favoring a complete combustion.

\subsubsection{Design}

No specific reported information has been found in the web pages of the two most well- known and/or available to buy devices. Drawings of the devices are shown in web pages for those available to buy without dimensions details. The Ethos MAXPower Super Fuel Unit is said to be powered by a Patented Neodymium Super Conductor which delivers a magnetic induction of 12,000 gauss and it is designed for the use of fuel lines up to $16 \mathrm{~mm}$ in diameter. Gauss is the standard SI unit of measurement for magnetic induction, also known as Magnetic Flux Density. In one of the Fuel Optimizer (efficient Argentinian device) web pages, it is emphasized that it is better than the other competitors' devices without specifying how much the magnetic induction of the magnets employed is. In similar patented devices, drawings with dimensions of parts are shown and a detailed functioning of the device as a whole is made but a complete and rational engineering design based mainly on physics and in chemistry is not shown either. An approach based on the engineering design of a magnetic fuel saver in physics may be found in the open literature ${ }^{2}$.

\subsubsection{Building}

The assembly of available devices in the current market seems to be not complicated, although there is no details of engineering design. There is little information available in the web pages, about building for commercialization.

\subsubsection{Installation}

These devices are installed in line with the fuel line, preferably close to the carburetor or the injection system without using any tool nor cutting the pipeline. Neither rare hoses nor fasteners are needed for installation. It is claimed that the miles per gallon is increased by $20 \%$ or more. When they are installed on the line that feeds the fuel into carburetor or injection, a better engine performance is obtained. This results in:

- More miles per gallon of fluids, reaching savings $20 \%$ and more of normal consumption

- Cleaner combustion chamber

- Considerable reduction of the emission of harmful gases

- Longer engine life due to less carbon formation and hydrocarbon contamination of engine oil, and

- The ease of installation

The number of devices to install depends on the engine capacity and the class of fuel. As an example, in the case of the optimizer for gas engines up to 2.5 liters, one device is needed. Those engines with larger than 2.5 liters, they needs two devices, whereas those with larger than seven liters, they need three devices.

\subsection{Devices Combining Magnetic Action and Fuel Hydraulic Flow Changes}

These devices are the combination of two processes to optimize combustion. These kinds of devices are not available in the current market, only they have been patented in US Patent Office and EPO. Two of such devices are described in 
this section. The first is a fluid saving device including a fuel multidirectional device in a magnetic field. The multidirectional device includes a reservoir and a plurality of spirals surrounding the reservoir for passage of the fuel in turbulent manner through a magnetic field. This turbulence splits the clusters of molecules of the fluid, ionize them and makes the magnetic field action to ionize the fuel easier because it breaks the cluster of molecules. This device was patented in USA in 1988, as Fuel saver; US Patent Number 5,673,674 ${ }^{3}$. The most important difference is that the magnetic field is provided using an electrical current, being this procedure not feasible neither technically nor economically for the vast majority of vehicles using internal combustion engines. Even if technically possible, it will not be economically feasible since with powerful small magnets, even higher magnetic fields can be obtained at a lower cost.

The second device is the one patented in USA in 1983, as magnetic device for treating hydrocarbon fuels; US Patent Number $4,372,852^{4}$. It is a magnetic device for treating hydrocarbon fuel, including a passageway having an inlet and an outlet for the passage of the hydrocarbon fuel, a pair of elongated magnets. Each magnet is magnetized to have one pole extending along one longitudinal face and the other pole extended along the opposite longitudinal face. The pair of magnets is located along and opposite sides of the passageway with the faces of the magnets having as poles spaced from and parallel to each other. The pair of magnets provide a substantially unipolar magnetic flux on fuel flowing in the passageway.

In order to emphasize in the importance of hydraulic fuel flow changes to ionize the fluid, a comparison is made with a device patented in US, Patent number $7,412,974^{5}$ belonging to especial kind of devices to improve the fuel efficiency of an internal combustion engine by means of hydraulic fuel flow changes. Generally, it includes a conical shaped member positioned in the gas flow generated by a motor. One or more deformations, such as slots, openings, v-cuts or other types are formed in the conical member to alter one or more characteristics, such as the pressure and velocity of the gas flow. The device can be positioned in the air intake system. Alternatively, the apparatus can be positioned in the exhaust system. The flow conditioners can also be of the type inserted into the fuel line to alter the characteristics of the fuel flow. These $s$ kinds of devices pretreat the fuel prior to the magnetic field action, which results in devices that are more efficient. This conjecture was proved by a simple but general experiment performed in December 22 1997, in Cartagena de Indias, Colombia.

\subsubsection{Design}

In all devices, the fuel efficiency of an IC depends mainly on the extent of ionization prior to combustion but using a pretreatment before the magnetic action imposing hydraulic fuel flow changes. This pretreatment imparting turbulence to the fuel may be achieved in many different ways resulting in a great variety of devices of different geometric arrangements and structures with different efficiencies. Neodymium magnets are not used in these devices.

\subsubsection{Building}

The structure and geometry of these devices are varied, ranging from very easy as the Magnetic device for treating hydrocarbon fuels to very complex geometries, passing for intermediate geometries as the Fuel saver. It is rare to find the class of construction materials and the details of dimensions in the patent web pages. These patented devices were efficient for the purpose of its invention but since the advent of super magnets as the neodymium ones, most of them only have a basic and reduced field of practical application.

\subsubsection{Installation}

For mobile sources as cars, motorcycles, and trucks, these devices are installed, when possible, in line with the fuel line preferably closest to the carburetor or the injection system. It is necessary to cut the fuel line. Hoses, fittings and fasteners are needed for installation. Difficulties for installation in cars and similar range from non-feasible technically and economically to easy installation, as the prototype or the Magnetic device for treating hydrocarbon fuels, as examples, passing through intermediate complexity installations. Details for installation and others may be found in the correspondent patent web pages of USPTO (United States Patents Office) 


\section{Advantages and Disadvantages of Magnetic Devices in Comparison with the Prototype}

After studying all the general information about magnetic minimizers with or without pretreatments and comparing the most efficient of both groups with the prototype, it has been found that they all have advantages and disadvantages depending on the purpose and circumstances. If the performance of devices is analyzed and its field of application is limited to mobile sources, according to the ADC results obtained and the testimony of users about the saving in fuel consumption, the Prototype (Minimizer) is the most efficient. However, because of higher costs in designing, building and installation, it is the most expensive device compared to all the devices using only magnetic action, though cheaper than the devices combining magnetic action and hydraulic fuel flow changes. Moreover, the manufacturing and installation of these magnetic action devices is easier than the Prototype and other patented magnetic devices. These patented devices that use magnetic action from permanent magnets or use an electric current are bigger in size, more expensive and more difficult to install than others are. However, these kinds of patented magnets are an empirical evidence that help to support the magnetic devices available in the market as well as those in the commercialization stage. On the other hand, those patented devices could be used in other circumstances different from mobile sources producing air pollution using IC engines, where the aforementioned magnetic devices are not so efficient or convenient to be used. Magnetic devices without pretreatment that are available in the market are claimed to have a great saving in fuel, which might not be true all the time. These devices do not mention any environmental impact in terms of air pollution produced during the combustion in engines. In addition, these Magnetic devices do not specify the percentages of emissions reductions. In the case of minimizer in the present study, it can be shown that the gas emissions are not unfavorably high and can be controlled by a proper engineering design.

\section{Conclusions about Efficiency of Magnetic Devices}

What device would be the most efficient under predetermined conditions would be concluded after a cost benefit analysis that must take into account the reduction of emissions that could be lethal with severe impact on the health and safety. In spite of the fact that magnetic devices could be optimized for fuel efficiency, there is a lot of controversy comments on their performance ${ }^{6}$.

There are several inconveniences about magnetic devices, especially those without pretreatment, as follows:

- They do not remain efficient under the continuous changes of mobile sources.

- Many of them have disappeared just after a short time of having an economical success.

- They do not say how to control effect of greenhouse gases emissions

- They do not have an engineering design to keep the device efficient under changes of the environment

- They are not improved continuously.

Despite all these drawbacks, these devices have helped to some extend in protecting environment by minimizing the amount of gas emission. Despite the use of these devices, there are millions of tons of polluting agents that are released to the atmosphere. It is clear that no device alone is so highly efficient to solve completely the problem of gas emissions or in a synergic connection with others. The increase of the automotive park in many countries is evident and the lack of renovation of the old one is still more. These factors in synergy with the new kinds of mobile sources, among other factors, keep on contributing enormously to worsen air pollution. On one hand, they increase the contaminants in air and on the other hand make the problem to get even worse due to inefficient current anti-pollution devices under exigencies of the new mobile sources. Consequently, they are brought to discredit, not able to be in demand, able to discourage even the fabricators themselves and finally entail an important reduction in number of devices to cut air-polluting emissions. Therefore, there is still a need in improvement of such devices to make them more efficient in terms of minimizing gas emissions. There are several questions that need to be clarifed:

- Why do many devices once efficient get discredited and then disappeared?

- Why do not devices remain efficient under the continuous changes of mobile sources?

- Why are not they improved continuously to face changes of work conditions?

- Why do not they control greenhouse gas emissions?

- Why do they emphasize more in fuel saving than in emission reduction to regulated limits? 
There is only one answer for all these questions: The economic interest has prevailed before the interest to reduce air pollution. All the inconveniences could be solved with an appropriate engineering design complemented with empirical information from ADC (Automotive diagnostic center) and the regulations of the country, region, province or state where the device is going to be used. Finally, we conclude that no efficient device must be discarded. Many efficient devices must be built to help to reduce air pollution but according to an adequate engineering design. This is the first step to fabricate efficient magnetic devices.

\section{Criteria for Designing, Building and Installing a Magnetic Device (Minimizer)}

\subsection{Design}

To make an efficient design to control gas emissions from mobile sources with a magnetic device, the following steps must be taken into account:

1. Identifying precisely the physical and/or chemical processes ruling the fuel ionization and how interact/complement to each other using correctly the laws of physics and chemistry that should be applied.

2. Identifying variables representing measurable and/or calculable physical fuel properties that finally could be related with the dimensions of the device to be designed.

3. Modeling of processes (setting up of an experimental assembly) in order to measure the variable properties.

4. Careful observing and reviewing the system performance and measurement

5. Interpreting experimental data

6. Magnetic induction of the magnets used

7. Type and composition of the gasoline

8. Knowing the gas emission standards of the country or region where the device will be used

9. Results obtained with the prototype in an ADC

10. Projected percentages of combustion products

11. To write all the equations needed to size the devices

12. Sizing the device.

\subsection{Building}

13. Equipment, tools and materials (couplings, hoses, fittings, etc.) to be used

\subsection{Installation}

14. Characteristics of the Mobile source Engine

15. Characteristics of the fuel supply system.

Design Steps 1-12

Proceeding in the order of listed steps, concerning the prototype, two physical processes working in favor of the fuel ionization are identified. These include a magnetic action on the clusters of molecules constituent of the fuel, caused by a couple of neodymium magnets, and a hydraulic action on the fuel molecules, caused by a section enlargement. These actions are complemented with a piece of an alloy metal with holes and grooves to increase the turbulence of the fluid so that the clusters of molecules break causing its own ionization in order to improve the magnetic action. The conjecture of the ionizing effect of the mentioned hydraulic action was proven in a simple experiment performed recently. According to that experiment, the variable that may be related to the dimensions of the device is the conductivity $\sigma$ and/ or its inverse the resistivity $\rho$. The magnetic induction to get an efficient magnetic action can be calculated through a quantum approach from known physical quantities ${ }^{7}$ but also from the energy required to get the amounts of projected combustion products. However, commercial magnets are fabricated with specific and multipurpose dimensions and magnetic inductions ${ }^{8}$. Experience shows that for a car or a van, magnets of 2300 gauss are enough. For heavier vehicles (buses and trucks), higher magnetic induction magnets should be used. Perhaps two or three couples of 2300 gauss magnets should be sufficient. Type and composition of the gasoline varies from country to country. A proven efficient magnetic device in a country might not be considered by other countries. Therefore, the current gas emission standards of the country or region 
should be known. These are very important to project the percentages of combustion products. The design of a magnetic gas emission reducer, in particular the chosen prototype (minimizer), is not as simple as a current engineering design could be. Gasoline is a refined product of petroleum consisting of a mixture of hydrocarbons, additives, and blending agents. The composition of gasolines varies widely, depending on the crude oils used, the refinery processes available, the overall balance of product demand, and the product specifications ${ }^{9}$. On the other hand, the changes by magnetic action with or without hydraulic pretreatments, at the level of fuel molecules, are very small; therefore, a quantum approach should be used. Although it is not impossible to size a magnetic device, it is difficult to size a device that is valid for many different circumstances. Rather, any design must be based on experimental results and supported by test results from ADC for typical conditions and/or experiments. With regard to the prototype (minimizer) or similar to be used for light vehicles (motorcycles, cars, vans and small trucks), the sizing is limited to test, in an ADC prototype devices of proven efficient dimensions and known magnetic induction magnets. In other cases, the design may be based on a quantum approach using the energy provided by two neodymium magnets of known magnetic induction for transforming hydrogen in the fuel from its diamagnetic state (stable) to paramagnetic (unstable state with higher energy) ${ }^{10}$. The global reduction of consumption is found, and then the rate of volume change with respect to energy is calculated. Finally, the dimensions of the device are calculated from this rate, assuming a circular constant section. The design may be also based on the ADC results and the global reduction of the consumption of fuel due to optimum combustion. This allows sizing the device assuming a constant circular section whose current diameters values range from $3 / 8$ to $3 / 4$ inches where only the length is necessary to calculate. Whichever experiments and procedures are used to size the device, the following procedure scheme is necessary to follows:

\section{Prototype Test $\rightarrow$ Initial Dimensioning $\rightarrow$ Adjustment Test $\rightarrow$ Final Dimensioning}

The following group of equations can be used to size the prototype (using quantum approach):

Equation 1 expresses variation of volume with respect to total provided energy to the gasoline sample

Equation 2 expresses the relationship between the initial volume (no energy provided) $k=$ constant

Equation 3 expresses total energy $E$ in terms of Avogadro Number and energy $E_{H}$ provided to a hydrogen atom. Equation 4 expresses energy of hydrogen atom in terms of the magnetic induction of the Neodymium magnets. Equation 5 expresses of the gasoline sample contained in the volume of the device.

$$
\left.\left.\left.\left.\left.\frac{\partial V}{\partial E}=k 1\right), k=\frac{V_{0}}{E} 2\right), E=13.5 N_{0} E_{H} 3\right), N_{0}=6.02 \times 10^{23} \mathrm{~mol}, E_{H}=F(B) 4\right), V=A L=\frac{\pi d^{2}}{4} l 5\right)
$$

\section{Building Step 13}

Although it is not so difficult to build the devices, a wide commercialization of the device is too expensive not only for the marketing but also due to the design and manufacturing the device based mostly on experiments, tests and control to produce an efficient device that may not be obsolete very soon.

\section{Installation Steps 14-15}

The device must be mounted on a short plastic tube coupled to the fuel supply line when this is meshed with a metallic cover. It is provided with a small compass inserted, externally, at the end of the device outputting the fuel, installed in line with the fuel conduit, in the same direction of the earth magnetic field, to control its magnetic action that in some instances could count rest the neodymium magnets action on the fuel.

\section{References}

[1] ETHOS, UK, (2018), Max Power. [Online]. Available: http://ethosplan.com/empsfuel.aspx

[2] M. Y. El Hafidi, M. El Hafidi, "Design and Performance Study of a Magnetic Fuel Saver, 3.1 Quantum Approach," Moroccan Journal of Condensed Matter, vol. 13, pp. 1-3, 2011. [Online]. Available: http://revues.imist.ma/index.php?journal=MJCM\&page=article\&op=view\&path\%5B $\% 5 \mathrm{D}=445$

[3] J. Monteiro. "Fuel saver." U.S. Patent Number 5,673,674, Oct. 7, 1997.

[4] "Magnetically device for treating Hydrocarbon fuels." U.S. Patent Number 4,372,852, Feb. 8, 1983. 
[5] R. B Russel. "Device for Enhancing Fuel Efficiency of IC Engines.” U.S. Patent Number 7,412,974.

[6] R. Guerrero, E. Quinones, Efficient Iofet Device To Cut Emissions From Mobile Sources, Slides 4-7, VII IV Congreso De Gestión Ambiental, Habana, July 6-10, 2009.

[7] M. Y. El Hafidi, M. El Hafidi, "Design and Performance Study of a Magnetic Fuel Saver," Moroccan Journal of Condensed Matter, vol. 13, p. 1, 2011. [Online]. Available: http://revues.imist.ma/index.php?journal=MJCM\&page=article\&op=view\&path\%5B \%5D=445

[8] DIMETALES, Bogotá Colombia, 2018. [Online]. Available: http://dimetales.com/home/productos/imanes-deneodymium

[9] Chemical Identity of Gasoline. [Online]. Available: https://www.atsdr.cdc.gov/toxprofiles/tp72-c3.pdf

[10] M. Y. El Hafidi, M. El Hafidi, "Design and Performance Study of a Magnetic Fuel Saver," Moroccan Journal of Condensed Matter, vol. 13, p. 1, 2011. [Online]. Available: http://revues.imist.ma/index.php?journal=MJCM\&page=article\&op=view\&path\%5B\%5D=445

[11] R. Guerrero and M. Mehrvar, "High Efficiency Device to Cut Gases Emissions from Mobile Sources," in RTESE'17, Toronto, Canada, 2017.

[12] R. Guerrero, "High Efficiency Device to Cut Gases Emissions from Mobile Sources," in RTESE'17, Toronto, Canada.

[13] ECONOPLUS, Argentina, 2004. [Online]. Available: http://www.fueloptimiser.com.ar

[14] USPTO USA, (February 12, 2018). [Online]. Available: https://www.uspto.gov

[15] USPTO USA, (February 12, 2018). [Online]. Available: http://patft.uspto.gov/netahtml/PTO/search- bool.html

[16] EPO, Europe, (February 12, 2018). [Online]. Available: https://lp.espacenet.com

[17] Análisis de la Combustión Completa e Incompleta. [Online]. Available: http://materias.fi.uba.ar/6730/Tomo1Unidad2.pdf

[18] I. Martínez, Fuel Properties, [Online]. (2018), Available: http://webserver.dmt.upm.es/ isidoro/bk3/c15/Fuel\%20properties.pdf

[19] Fuel Properties, Budapest University of Technology and Economics. [Online]. Available: ftp://ftp.energia.bme.hu/pub/AviationEngineerSpecialist/PowerPlant/AvEng_Fuelproperties.pdf

[20] Colombia, Ministerio del Medio Ambiente, Resolución, 1111 (September 2013). [Online]. Available: http://www.minambiente.gov.co/images/AsuntosambientalesySectorialyUrbana/pdf/emisiones_atmosfericas_contam inantes/fuentes_moviles/Resolución_1111_de_2013_-Modifica_parcialmente_Resolución_910_de_2008.pdf

[21] Regulation of Air Pollution in Canada. [Online]. Available: http://www.oagbvg.gc.ca/internet/English/pet_055_e_28762.html\#Ambientstd

[22] J. E. Brady and G. E. Humiston, General Chemistry Principles and Structure. USA, 1982.

[23] E. M. Purcell, "Chapter 10 Magnetic Fields in Matter," in Electricity and Magnetism. Harvard University, McGraw Hill, 1982, pp. 360-408,

[24] R. Feynman, R. B. Leighton, M. Sands, “Chapter 34,” in Physics, vol. 2. USA, 1987.

[25] J. Binney, Quantum Mechanic Lectures 12, 18, 19, 20, 22-24, University of Oxford, Dec. 2009. 